\title{
Influência do meio (urbano e rural) no padrão de aptidão física de rapazes de Viana do Castelo. Portugal
}

\author{
Luís P. Rodrigues \\ Pedro Bezerra \\ Linda Saraiva
}

https://doi.org/10.5628/rpcd.05.01.77

\section{RESUMO}

Nas últimas décadas temos vindo a assistir a alterações profundas nos estilos de vida da população infanto-juvenil. Associada a um crescente grau de urbanização, a sedentarização parece promover o declínio dos níveis de aptidão física das crianças e jovens. Os meios ruralizados são usualmente relacionados com estilos de vida mais vigorosos, no entanto a investigação desta assumpção nesta faixa etária tem sido escassa ou com conclusões algo contraditórias. O objectivo deste trabalho foi comparar os padrões de aptidão física de rapazes (7 aos 10 anos) vivendo em contextos envolventes diferenciados (urbano / rural). Foram analisados 1832 rapazes, pertencendo à base de dados do Estudo Morfofuncional da Criança Vianense. Os efeitos da Idade e do Meio no comportamento da aptidão física foram testados através de técnicas multivariadas e univariadas. Os resultados permitiram concluir que: (i) a aptidão física melhorou significativamente de ano para ano, independentemente do meio; (ii) os rapazes dos dois grupos exibiram uma estrutura significativamente diferente de aptidão física ao longo das idades; (iii) os rapazes das zonas rurais demonstraram ser consistentemente superiores nas provas de Pull Up, Corrida de 50 metros e Corrida de Vaivém, enquanto os seus pares do meio urbano se evidenciaram nas prestações de Shuttle Run e Salto Horizontal.

Palavras-chave: aptidão física, crianças, rapazes, urbano/rural, estilos de vida.

\author{
Instituto Politécnico de Viana do Castelo \\ Escola Superior de Educação \\ Portugal
}

\begin{abstract}
Environmental (urban-rural) influences on physical fitness pattern of 7 to 10 year-old boys in Viana do Castelo, Portugal.

Children and youth lifestyles are rapidly changing. Along with a general decrease in physical activity, an increased urbanization of the living spaces can be responsible for a noted decline in physical fitness.

Although rural spaces are usually assumed to be related to more vigorous lifestyles, minimal information regarding school-aged children is available in the literature. The goal of this study was to compare fitness levels of elementary school rural and urban boys. The sample was drawn from the Estudo Morfofuncional da Criança Vianense database. Multivariate and univariate statistics were used to clarify the effects of the Environment (urban / rural), and Age (7-to-10 years-ofage). The results showed that: (i) physical fitness improved along all ages, independent of the environment; (ii) rural and urban boys revealed significantly different physical fitness profiles; (iii) rural boys performed significantly better on the Pull Up, 50 meter Dash and 20-meter Pacer Run, while their urban counterparts showed superior results on the Shuttle Run and Standing Long Jump.
\end{abstract}

Key Words: physical fitness, children, boys, urban/rural, lifestyle. 


\section{INTRODUÇÃO}

Nas últimas décadas temos vindo a assistir a alterações profundas nos hábitos de vida da população infanto-juvenil dos países ocidentais. Associada a um crescente grau de urbanização, a sedentarização dos estilos de vida parece promover o aparecimento de problemas associados à saúde e bem-estar, nomeadamente o aumento da obesidade (10) e a diminuição dos níveis de aptidão física $(\mathrm{ApF})$ das crianças e jovens $(7,13)$. São inúmeras as razões evocadas para explicar este fenómeno, nomeadamente as condições mais compactas da vida urbana com a consequente degradação das condições e oportunidades para actividade livre (24), o decréscimo da importância dos espaços de convívio, jogo e aventura (19), e a redução da autonomia de mobilidade no quotidiano das crianças do meio urbano $(3,20)$. A residência em meios menos urbanizados é usualmente associada a estilos de vida mais vigorosos, resultando em melhores desempenhos motores e níveis superiores de aptidão física. De facto, quando a ApF foi analisada na sua estrutura multivariada, ou seja comparando não os testes um a um mas o seu conjunto em simultâneo, foi possível encontrar diferenças significativas na expressão da $\mathrm{ApF}$ de rapazes entre os 6-9 e os 10-13 anos de idade da província de Oaxaca, no México (22). De igual modo no distrito de Coimbra, adolescentes entre os 14-16 anos provaram ser significativamente influenciados pelo grau de urbanização do seu meio envolvente (27). No entanto, a produção de investigação que permita clarificar melhor estas diferenças tem sido particularmente escassa na faixa etária entre os 7-10 anos, e quando existente as conclusões revelam-se algo contraditórias. Por exemplo, em Portugal conseguimos encontrar estudos conferindo vantagem significativa às crianças do meio rural nas provas de lançamento em distância (25), dinamometria manual e de agilidade (18), mas é também nesta última prova que as crianças do meio urbano da Guarda (25) e Castelo Branco (21) se demonstraram superiores aos seus pares do meio rural. O panorama internacional não se revela menos confuso. Exemplo típico é o da aptidão cardiorespiratória, em que valores superiores têm sido atribuídos quer a crianças do meio rural (8, $9,29)$, quer às do meio urbano $(17,23,28)$.

Também na velocidade, rapazes mexicanos (22) entre os 6-9 anos de idade do meio rural foram mais rápidos que os seus pares do meio urbano, mas esta diferença esbateu-se rapidamente no nível etário seguinte (10-13 anos).
A nosso ver, algum desconcerto face a estes resultados é compreensível dado: (i) a indefinição na caracterização dos meios contrastados, (ii) a multivariedade cultural de povos e/ou regiões, (iii) a evolução social e económica das últimas décadas que resultou em rápidas alterações dos ambientes em análise, (iv) a necessidade, nem sempre contemplada, de isolar a $\mathrm{ApF}$ das características morfológicas e maturacionais, e (v) a falta de uniformidade nas técnicas e instrumentos utilizados para avaliar o constructo da ApF. Perante estas tendências contraditórias e uma conjuntura nacional de crescente urbanização territorial, parece-nos pertinente perceber quais os seus possíveis efeitos nos níveis de ApF das nossas crianças. Será que os estilos de vida adoptados nos meios mais urbanizados podem constituir uma influência negativa na expressão da ApF das crianças? Será possível identificar distintos padrões de ApF em função do grau de urbanização em que vivem as nossas crianças? Estas são as perguntas que procurámos responder neste estudo onde contrastamos a ApF de crianças do sexo masculino vivendo no meio urbano com os seus pares que habitam espaços mais ruralizados. Porque foi nossa pretensão que a unidade principal de análise fosse a ApF como constructo latente e individualizado dos vários testes marcadores utilizados, decidimos recorrer a técnicas estatísticas multivariadas que nos permitissem comparar o padrão multidimensional da ApF das crianças do $1^{\circ}$ Ciclo do Ensino Básico dos meios urbano e rural no distrito de Viana do Castelo. Necessário será ainda esclarecer que o nosso entendimento da interacção entre ApF, Saúde e Bem-estar, não se resume nas variáveis em que é possível determinar uma relação directa (como é o caso da aptidão cardiovascular ou da adiposidade). A expressão da ApF na sua associação mais clássica à performance parece-nos ainda um instrumento útil e adequado, quer à nossa população (portuguesa e vianense), quer especialmente às idades escolares mais baixas. Nas crianças e jovens, os níveis de ApF não devem ser encarados somente como resultados de vivências anteriores, mas também como elemento despoletador de estilos de vida futuros. Crianças mais aptas e com melhores facilidades de desempenho tenderão a envolverse mais em práticas de actividade física e a sentirem-se mais recompensadas. É no âmbito deste efeito multiplicador indirecto dos níveis e/ou padrões de ApF e suas implicações nos estilos de vida que inserimos esta nossa investigação, num momento em que a actividade física se apresenta 
cada vez mais como o denominador comum para a compreensão da relação entre a aptidão física, a obesidade e a saúde $(4,5,14)$.

\section{MATERIAL E MÉTODOS}

\section{Amostra}

A amostra utilizada neste artigo e que é descrita em pormenor no Quadro 1, pertenceu ao Estudo Morfofuncional da Criança Vianense (EMCV), investigação que decorreu de 1996 a 2000 no concelho de Viana do Castelo e que recolheu dados morfológicos, bio-sociais e de aptidão física de crianças do $1^{\circ}$ Ciclo do Ensino Básico. A escolha das quinzes escolas pertencentes ao EMCV obedeceu a critérios de localização geográfica e representatividade equitativa de idades, género e ambiente sócio-económico. As duas escolas do meio urbano, mais populosas, situavamse no centro da cidade de Viana do Castelo e pertenciam a freguesias consideradas exclusivamente como urbanas segundo o critério de classificação territorial do INE (11). As restantes treze escolas encontravam-se dispersas no tecido ruralizado do Concelho.

Quadro 1. Distribuição da amostra em função do Meio (urbano-rural), Escolas e ldade.

\begin{tabular}{|c|c|c|c|c|c|}
\hline \multirow[b]{2}{*}{ Escolas } & \multicolumn{4}{|c|}{ Idade } & \multirow[b]{2}{*}{ Totais } \\
\hline & $?$ anos & 8 anos & 9 anos & $\begin{array}{c}10 \\
\text { anos }\end{array}$ & \\
\hline Meio Urbano & 212 & 234 & 232 & 215 & 893 \\
\hline Avenida & 75 & 72 & 84 & 88 & 319 \\
\hline Carmo & 137 & 162 & 148 & 127 & 574 \\
\hline Meio Rural & 236 & 239 & 235 & 229 & 939 \\
\hline Portelas & 37 & 37 & 38 & 34 & 146 \\
\hline S. Gil & 20 & 20 & 23 & 25 & 88 \\
\hline Cardielos & 28 & 29 & 32 & 32 & 121 \\
\hline Outeiro & 10 & 13 & 12 & 15 & 50 \\
\hline Nogueira & 17 & 15 & 14 & 15 & 61 \\
\hline Serreleis & 23 & 24 & 21 & 15 & 83 \\
\hline Samonde & 18 & 16 & 9 & 6 & 49 \\
\hline Deocriste & 14 & 9 & $?$ & 8 & 38 \\
\hline Sta M. Geraz & 11 & 15 & 19 & 18 & 63 \\
\hline \multicolumn{6}{|l|}{ Lima } \\
\hline S. Salvador & 5 & 8 & 10 & 10 & 33 \\
\hline Subportela & 20 & 21 & 21 & 22 & 84 \\
\hline Vila Mou & 11 & 13 & 9 & 9 & 42 \\
\hline Deão & 22 & 19 & 20 & 20 & 81 \\
\hline Totais & 448 & 473 & 467 & 444 & 1832 \\
\hline
\end{tabular}

\section{Variáveis}

A selecção dos testes de ApF utilizados no EMCV obedeceu a critérios de familiaridade, possibilidade de utilização ao longo de sucessivos níveis etários, e facilidade de administração e medição/avaliação das várias componentes da aptidão física com pouco ou nenhum equipamento. Para além disso, e pelas razões aduzidas anteriormente na introdução, foi nossa intenção explorar o registo multifacetado de desempenho da ApF. Como resultado, as variáveis de ApF recolhidas foram o sit-and-reach (SR), o número de abdominais em 60 segundos com pernas flectidas e braços cruzados (ABD), o salto em comprimento sem corrida preparatória (SCP), o tempo máximo de suspensão na barra (TSB), o shuttle-run em 10 metros (SHR), a corrida de velocidade em 50 metros (C50) e a corrida de resistência em vaivém de 20 metros $(\mathrm{CVV})$. Os seis primeiros testes pertencem à bateria da AAHPERD Youth Fitness (1) e à AAHPERD Health Related Physical Fitness (2), e o último à bateria de testes Eurofit (6). No decurso deste artigo serão ainda utilizadas duas das variáveis morfológicas recolhidas no EMCV, a altura e peso. A sua mensuração obedeceu ao protocolo descrito por Lohman, Roche e Martorell (15).

\section{Recolha de dados}

A recolha de dados foi inteiramente conduzida nas instalações da Escola Superior de Educação de Viana do Castelo e decorreu anualmente durante os meses de Abril e Maio dos últimos quatro anos (19972000) do EMCV. Após terem sido obtidas autorizações do Centro de Área Educativa de Viana do Castelo e dos pais das crianças, os alunos das escolas pertencentes ao estudo deslocaram-se com o apoio de autocarros pertencentes à Câmara Municipal de Viana do Castelo e, durante uma manhã, cada criança percorreu um circuito de mensuração morfológica e testagem da ApF. O circuito de ApF decorreu num ginásio e espaço desportivo exterior e a sua ordem de execução foi: TSB, SR, SHR, SCP, ABD, C50 e CVV. Todos os procedimentos utilizados no EMCV respeitaram as normas internacionais de experimentação com humanos, expressas na Declaração de Helsínquia de 1975. 


\section{Controlo de qualidade dos dados}

A execução dos testes obedeceu aos protocolos descritos nas baterias de teste de onde são originários. No caso da CVV, cada grupo de testagem foi sempre acompanhado de um elemento adulto que ajudava a marcar o ritmo de corrida. Esta opção foi tomada para garantir a compreensão do teste, dada a sua novidade e as idades baixas testadas. Na C50 e SHR foram sempre registados dois tempos, sendo a média considerada como resultado final.

As equipas de observação foram constituídas por alunos finalistas do Curso de Educação Física, previamente treinados nas tarefas específicas que desempenhavam. Os observadores permaneceram fixos numa das estações de testagem durante toda a fase de recolha de dados. Uma em cada doze crianças foi escolhida aleatoriamente para repetir a execução dos testes (à excepção dos ABD e CVV, devido à sua natureza). Os coeficientes de correlação intra-classe (26) resultantes desta repetição são apresentados no Quadro 2. Todos os momentos da recolha de dados foram supervisionados pelo menos por um dos autores, de forma a assegurar a qualidade do processo. Os dados finais, após introdução numa base de dados informatizada, foram submetidos a um processo de detecção de erros. O registo de distribuição de cada variável foi analisado e todos os valores detectados como extremos foram reconfirmados nos registos originais e corrigidos ou apagados (nos casos em que existia erro evidente no registo original). Não foi utilizado qualquer tipo de substituição de valores em falta (missing values), pelo que todos os indivíduos a quem faltavam algum dos valores das variáveis em estudo foram retirados da amostra.

\begin{tabular}{lccccc}
\multicolumn{7}{c}{$\begin{array}{c}\text { Quadro 2. Valores do Coeficiente de Correlação Intraclasse } \\
\text { para cada variável por ano de recolha e total agregado. }\end{array}$} \\
\cline { 2 - 7 } & \multicolumn{5}{c}{ Anos de recolha } \\
\cline { 2 - 6 } Variáveis & 1997 & 1998 & 1999 & 2000 & $1997-2000$ \\
\hline & $(n=67)$ & $(n=55)$ & $(n=65)$ & $(n=61)$ & $(n=248)$ \\
TSB & .76 & .58 & .87 & .72 & .76 \\
SR & .93 & .93 & .91 & .89 & .92 \\
SHR & .87 & .74 & .90 & .91 & .86 \\
SCP & .92 & .95 & .92 & .94 & .93 \\
C50 & .84 & .80 & .79 & .80 & .81 \\
Alt & .99 & .96 & .99 & .99 & .98 \\
Peso & .99 & .99 & .98 & .99 & .99 \\
\hline
\end{tabular}

Nota: Os CCl indicados são de tipo 3,1 (26), geralmente referidos como medidas simples de correlação intraclasse.

\section{Procedimentos estatísticos}

Os efeitos do Meio (urbano e rural) e da Idade (7, 8, 9 e 10 anos) na estrutura compósita da ApF das crianças foram testados através de uma comparação multivariada, controlando para a altura e peso dos participantes (MANCOVA). A aplicação desta técnica estatística multivariada permitiu preservar a relação entre cada variável recolhida, representando assim mais fielmente a estrutura complexa do padrão de ApF dos indivíduos. A decisão de utilizar as duas variáveis morfológicas como covariantes é um procedimento comum neste tipo de análise (16, 22) e decorreu de se ter encontrado uma associação significativa entre elas e os resultados da ApF expressos por idade e meio (12). Procurou-se assim que eventuais diferenças morfológicas entre os indivíduos dos grupos analisados não influenciassem na expressão das componentes de ApF estudadas. Análises de cada variável (ANCOVAs) foram também posteriormente utilizadas para melhor esclarecer o sentido das diferenças encontradas na comparação multivariada. O nível de confiança foi mantido em .05 para todas as análises estatísticas e o software utilizado foi o SPSS 11.0.

Como nota metodológica convém esclarecer que, apesar da verificação dos pressupostos de normalidade dos dados ter detectado desvios em algumas das variáveis (com destaque para o TSB), a realização prévia das análises efectuadas com dados transformados pela técnica de Box-Cox permitiu confirmar que a elevada dimensão da amostra constituía garante suficiente da robustez das técnicas estatísticas utilizadas. Assim sendo, optámos pela apresentação dos resultados sem transformações, já que permitem uma mais fácil interpretação da realidade.

\section{RESULTADOS}

A análise multivariada demonstrou a expressão de efeitos significativos do Meio $\left(\mathrm{F}_{(7,1816)}=16.22\right.$; $p=.000)$ e da Idade $\left(\mathrm{F}_{(21,5215,1)}=24.09 ; p=.000\right)$, pelo que, e dada a não existência de interacção entre estes mesmos factores $\left(\mathrm{F}_{(21,5215,1)}=.671 ; p=.865\right)$, pudemos concluir pela independência e aditividade dos efeitos da Idade e do Meio na estrutura da ApF destas crianças.

Procurando especificar melhor estas diferenças de estrutura, procedemos à análise de comparação uni- 
variada nas componentes de ApF testadas. No Quadro 3 são apresentados os resultados descritivos (médias e desvios-padrão dos valores reais) para cada variável, bem como os resultados da comparação efectuada após ajustamento às covariáveis (ANCOVAs). Desta última análise, em que a influência do peso e altura da crianças no desempenho dos testes de ApF foi controlada, verificou-se que subsiste um efeito generalizado e significativo da Idade para todas as variáveis testadas $(p=.000)$ com excepção do SR $(p=.46)$. Quer isto dizer que, e em todas as provas à excepção do SR, se verificou uma melhoria significativa das prestações, de ano para ano, em qualquer um dos dois grupos em análise.

Quadro 3. Valores descritivos (médias e desvios-padrão) dos testes de aptidão física e variáveis morfológicas dos rapazes do Meio Urbano e Rural e resultados dos efeitos da Idade e Meio [após controlo para as medidas morfológicas] para cada teste.

\begin{tabular}{|c|c|c|c|c|c|c|c|c|}
\hline \multirow[b]{2}{*}{ Var } & \multirow[b]{2}{*}{ Meio } & \multicolumn{4}{|c|}{ Idades } & \multicolumn{3}{|c|}{ Resultados ANCOVA } \\
\hline & & $?$ & 8 & 9 & 10 & $\begin{array}{l}\text { Idade } \\
F_{[3,1822)}\end{array}$ & $\begin{array}{c}\text { Meio } \\
F_{[1,1822]}\end{array}$ & $\begin{array}{l}\text { Id X Meio } \\
F_{(3,1822)}\end{array}$ \\
\hline \multirow[t]{2}{*}{ TSB } & Urb & $14.9 \pm 13.4$ & $16.5 \pm 13 . ?$ & $19.6 \pm 15.2$ & $20.7 \pm 15.4$ & 26.3 & 7.0 & 1.09 \\
\hline & Rur & $19.1 \pm 17.4$ & $18.7 \pm 15.4$ & $19.8 \pm 16.4$ & $22.9 \pm 17.9$ & $(p=.00)$ & $(p=.00)$ & $(p=.35)$ \\
\hline \multirow[t]{2}{*}{ SR } & Urb & $26.2 \pm 5.5$ & $25.1 \pm 5.9$ & $24.5 \pm 6.0$ & $24.2 \pm 6.5$ & 0.85 & 1.14 & 0.70 \\
\hline & Rur & $25.2 \pm 5.8$ & $24.8 \pm 7.7$ & $27.0 \pm 7.4$ & $29.7 \pm 8.3$ & $(p=.46)$ & $(p=.28)$ & $(p=.55)$ \\
\hline \multirow[t]{2}{*}{ SHR } & Urb & $13.3 \pm 1.2$ & $12.6 \pm 0.9$ & $12.0 \pm 0.8$ & $11.6 \pm 0.7$ & 121.9 & 14.6 & 0.69 \\
\hline & Rur & $13.3 \pm 1.1$ & $12.8 \pm 1.0$ & $12.2 \pm 0.9$ & $11.8 \pm 0.9$ & $(p=.00)$ & $(p=.00)$ & $(p=.55)$ \\
\hline \multirow[t]{2}{*}{ SCP } & Urb & $103.9 \pm 16.1$ & $115.7 \pm 18.6$ & $125.0 \pm 18.6$ & $135.3 \pm 19.1$ & 84.0 & 30.3 & 0.21 \\
\hline & Rur & $101.3 \pm 16.8$ & $112.4 \pm 18.0$ & $122.1 \pm 18.6$ & $131.0 \pm 19.0$ & $(p=.00)$ & $(p=.00)$ & $(p=.88)$ \\
\hline \multirow[t]{2}{*}{$A B D$} & Urb & $22.0 \pm 7.7$ & $25.3 \pm 7.7$ & $27.0 \pm 7.4$ & $29.7 \pm 8.3$ & 46.5 & 0.35 & 0.89 \\
\hline & Rur & $22.0 \pm 7.6$ & $25.8 \pm 7.9$ & $27.3 \pm 7.5$ & $28.7 \pm 7.6$ & $(p=.00)$ & $(p=.55)$ & $(p=.44)$ \\
\hline \multirow[t]{2}{*}{$\mathrm{C} 50$} & Urb & $11.6 \pm 1.1$ & $10.8 \pm 0.9$ & $10.2 \pm 0.9$ & $9.9 \pm 0.8$ & 114.9 & 4.1 & 0.21 \\
\hline & Rur & $11.4 \pm 0.9$ & $10.7 \pm 0.9$ & $10.1 \pm 0.8$ & $9.8 \pm 0.8$ & $(p=.00)$ & $(p=.04)$ & $(p=.88)$ \\
\hline \multirow[t]{2}{*}{ CVV } & Urb & $22.6 \pm 12.3$ & $28.6 \pm 14.4$ & $35.8 \pm 16.0$ & $41.3 \pm 18.5$ & 83.9 & 17.6 & 0.46 \\
\hline & Rur & $25.9 \pm 12.3$ & $32.6 \pm 13.8$ & $39.1 \pm 16.2$ & $44.5 \pm 18.3$ & $(p=.00)$ & $(p=, 00)$ & $(p=.88)$ \\
\hline \multirow[t]{2}{*}{ Alt } & Urb & $122.1 \pm 5.2$ & $127.5 \pm 5.8$ & $132.5 \pm 6.1$ & $137.7 \pm 6.1$ & & & \\
\hline & Rur & $122.1 \pm 5.0$ & $127.9 \pm 5.3$ & $133.6 \pm 5.7$ & $138.4 \pm 5.9$ & & & \\
\hline \multirow[t]{2}{*}{ Peso } & Urb & $25.4 \pm 4.9$ & $28.5 \pm 5.9$ & $31.5 \pm 6.4$ & $35.1 \pm 6.9$ & & & \\
\hline & Rur & $24.9 \pm 4.3$ & $28.2 \pm 5.5$ & $31.7 \pm 6.4$ & $35.0 \pm 7.3$ & & & \\
\hline
\end{tabular}

Nota: na apresentação dos valores descritivos das variáveis são apresentados os valores reais, ou seja sem ajustamento ao peso e altura dos indivíduos, para mais fácil interpretação do leitor. No entanto, os resultados das ANCOVAs expressos na parte direita do quadro são relativos à comparação após ajustamento às covariáveis peso e altura.

Por sua vez, o Meio demonstrou efeitos significativos nas provas de TSB, SHR, SCP, C50 e CVV, não lhe tendo sido possível atribuir efeitos diferenciadores nas variáveis $\operatorname{SR}(p=.28)$ e $\operatorname{ABD}(p=.55)$. A direcção dos efeitos encontrados não foi, no entanto, comum a todas as variáveis, como se comprova pelas imagens de comparação gráfica entre as médias (ajustadas ao peso e altura) das prestações dos dois grupos ao longo da idade (Figura 1). Assim, os rapazes do meio rural foram consistentemente mais proficientes nas provas de TSB, C50 e CVV, enquanto os rapazes do meio urbano demonstraram superioridade nas provas de SHR e SCP. Os dois grupos não demonstraram desempenhos significativamente diferenciados nas provas de SR e ABD. Não deixa de ser curioso referir que (mais uma vez com excepção do $\mathrm{SR}$ ), ambos os grupos conservam traçados relativamente paralelos na evolução do desempenho das provas de ApF ao longo dos quatro escalões etários. Este facto permite facilmente inferir da estabilidade das diferenças atribuíveis ao Meio entre os dois grupos. 

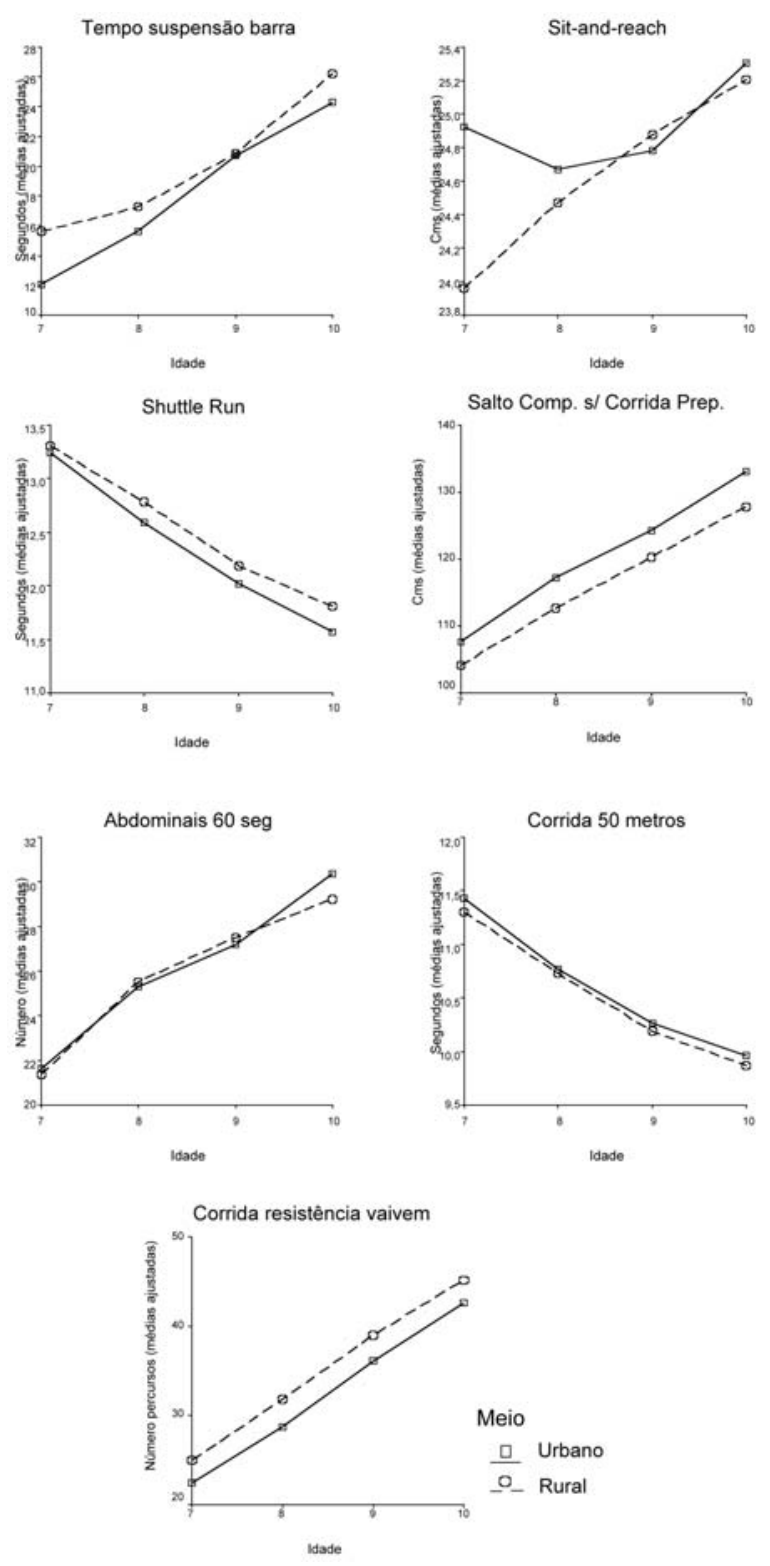

Figura 1. Representação gráfica das médias (ajustadas ao peso e altura) dos testes de aptidão física dos rapazes do meio urbano e rural, dos 7 aos 10 anos de idade.

\section{DISCUSSÃO}

O facto de a análise multivariada ter demonstrado efeitos significativos da Idade e Meio, mas não da interacção entre estes factores, permite-nos concluir que os rapazes do meio urbano e rural exibiram uma estrutura significativamente diferente na expressão da sua aptidão física ao longo das idades testadas. Resultados idênticos haviam sido encontrados em adolescentes do distrito de Coimbra (27) e rapazes mexicanos (22). A diferença persistiu mesmo após termos controlado os efeitos das variáveis morfológicas (peso e altura) que poderiam influenciar os resultados dos testes, acentuando assim a relevância do meio e dos estilos de vida a ele associados na construção do perfil de ApF destes rapazes. Esta evidência não permite, no entanto, realizar qualquer valoração da diferença encontrada, já que ela se situa no domínio das relações individuais entre os resultados dos testes utilizados. Por outras palavras, não podemos dizer que um dos grupos exibiu melhor (ou maior) ApF, apenas que os seus perfis foram efectivamente distintos. Na tentativa de perceber onde se situam essas diferenças de padrão (e apesar de corrermos o risco de alguma perca de informação na complexidade da expressão latente da $\mathrm{ApF}$ ), resolvemos analisar os resultados relativos a cada uma das variáveis marcadoras usadas. Dos resultados já referidos (Quadro 3) concluise, com facilidade, que a Idade é factor marcante na evolução das prestações de ApF, exprimindo-se na melhoria significativa das performances ao longo da idade na grande maioria dos testes (ABD, SCP, TSB, SHR, C50, CVV), sendo o comportamento peculiar do SR a única excepção encontrada. Nesta prova, a tendência para a diminuição da flexibilidade do tronco ao longo da idade parece evidente pela análise dos resultados médios encontrados, no entanto, quando controlamos os efeitos da altura e peso encontramos uma nítida inversão dessa tendência (Figura 1), com os rapazes do meio rural a melhorarem sempre as suas prestações relativas e os do meio urbano a fornecerem indicações de melhoria a partir dos oito anos.

Apesar de esperado, é sempre reconfortante verificar que o perfil de ApF de ambos os grupos evidenciou melhorias generalizadas com a idade. Já quanto aos efeitos do Meio, a expressão dos resultados nas diferentes variáveis permite caracterizar os distintos padrões de ApF detectados na MANCOVA e que estarão, provavelmente, relacionados com estilos de vida próprios do ambiente em que vivem: (i) o perfil dos rapazes do meio rural distinguindo-se nas pres- 
tações de força superior (TSB), corrida de velocidade (C50) e de resistência cardiovascular (CVV); e (ii) o dos rapazes do meio urbano, que se destacou nos testes de agilidade (SHR) e força inferior (SCP). Não sendo possível esclarecer completamente as razões para tais diferenciações, não deixa de ser curioso referir uma possível lógica aglutinadora para este fenómeno, já que os rapazes do meio rural apresentaram vantagem em provas associadas com características energéticas ou condicionais (C50, CVV, TSB), enquanto os seus pares do meio urbano se evidenciaram em situações onde a vertente coordenativa ou de execução do movimento poderá ser mais determinante (SHR e SCP). O que nos parece evidente, pela consistência dos resultados e pela dimensão e robustez do estudo, é que as condições de vida propiciadas a estas crianças se repercutiram de forma clara na expressão da sua ApF e, possivelmente de uma forma mais ampla, na sua motricidade. Apesar de nestas idades as diferenças encontradas não corporizarem indícios de alarme, os seus efeitos na adopção dos futuros estilos de vida destas crianças permanecem por avaliar. O maior ou menor acesso a instalações e/ou momentos de prática sistematizada, os ambientes sócio-económicos, os ritmos e espaços de vida, as rotinas de deslocação e actividade, a maior ou menor familiarização com procedimentos de testagem, ou mesmo uma expressão mais complexa das divergências culturais e exigências familiares próprias destes dois espaços (urbano e rural), poderão corporizar algumas das razões aparentes para as diferenças encontradas. Sugerimos, portanto, que a demanda futura desta questão possa passar pela configuração de modelos mais complexos de interacção entre as variáveis de ApF, as de crescimento e as de âmbito mais bio-social e motivacional. Em resumo, e respondendo às nossas próprias inquietações iniciais, pudemos comprovar que os rapazes vianenses vivendo em meios diferenciados segundo o grau de urbanização exibiram padrões de ApF significativamente distintos, sendo estas diferenças independentes da morfologia (altura e peso) exibida. Os rapazes que vivenciaram uma realidade menos urbanizada demonstraram melhores qualidades de força superior, velocidade e de resistência aeróbia, enquanto os rapazes do meio urbano se superiorizaram nas prestações de agilidade e força inferior.

\section{Agradecimentos}

Este estudo foi parcialmente apresentado no VI Congresso Nacional de Educação Física, organizado pela SPEF em Novembro de 2003, sendo de inteira justiça agradecer as contribuições que nessa ocasião foram feitas para a melhoria da redacção final deste trabalho. Os autores querem ainda expressar os seus mais profundos agradecimentos à Câmara Municipal de Viana do Castelo pelo apoio prestado, às escolas do $1^{\circ} \mathrm{CEB}$ e respectivos professores e alunos pela sua colaboração desinteressada, e aos alunos finalistas do Curso de Educação Física que constituíram a equipa de observação e sem os quais este estudo teria sido impossível de concretizar.

\section{CORRESPONDÊNCIA}

\section{Luís Paulo Rodrigues}

Escola Superior Educação Viana do Castelo

Av. Capitão Gaspar de Castro - Apartado 513

4901-908 Viana do Castelo

Portugal

lprodrigues@ese.ipvc.pt 


\section{BIBLIOGRAFIA}

1. AAHPERD (1976). Youth Fitness Test Manual. Washington, DC.

2. AAHPERD (1980). Health Related Physical Fitness Manual. Washington, DC.

3. Arez A, Neto C (1999). The study of independent mobility and perception of the physical environment in rural and urban children. In C. Neto (Ed.). Play and Community Proceedings of XIV 1999 IPA World Conference "The Community of Play". Lisboa: Edições C.M.L.

4. Blair S, Church T (2004). The fitness, obesity, and health equation. Is physical activity the common denominator? JAMA 292 (8): 1232-1234.

5. Brage S, Wedderkopp N, Ekelund U, Franks P, Wareham N, Andersen L, Froberg K (2004). Objectively measured physical activity correlates with indices of insulin resistance in Danish children. Int J Obesity 28 (11): 1503-1508.

6. Comitato Olimpico Nazionale Italiano (1988). EUROFIT Handbook. Rome.

7. Corbin C, Pangrazi R (1992). Are American children and youth fit? Res Q Ex Sport 63 (2): 96-106.

8. Dollman J, Norton K, Tucker G (2002). Anthropometry, fitness and physical activity of urban and rural south Australian children. Pediatr Exerc Sci 14: 297-312.

9. Ewing B (1982). The physical fitness and anthropometry of Scottish schoolboys - part 3. Scottish Journal of Physical Education 10 (1): 14-20.

10. Hedley A, Ogden C, Johnson C, Carrol M, Curtin L, Flegal K (2004). Prevalence of overweight and obesity among US children, adolescents, and adults, 1999-2002. JAMA 291 (23): 2847-2850.

11. INE (2003). Retrato territorial por NUTS e Concelhos. Retrieved July 13, 2004 from http://www.ine.pt/prodserv/retrato/retrato.asp.

12. Johnson R, Wichern D (2002). Applied Multivariate Statistical Analysis (5 $5^{\text {th }}$ ed.). New Jersey: Prentice Hall.

13. Kuntzleman C, Reiff G (1992). The decline in American children's fitness levels. Res Q Ex Sport 63 (2): 107-111.

14. Lederman S, Akabas S, Moore B (2004). Editor's overview of the conference on preventing childhood obesity. Pediatrics 114 (4): 1139-1145.

15. Lohman T, Roche A, Martorell R (1988). Anthropometric Standardization Reference Manual. Champaign: Human Kinetics Books.

16. Maia J, Prista A, Marques A, Lopes V, Saranga, S (2002). Estudo univariado e multivariado dos níveis de aptidão física. Efeitos da maturação biológica, do estatuto sócioeconómico e da percentagem de gordura corporal. In A Prista, J Maia, S Saranga, A. Marques (Eds). Saúde, crescimento e desenvolvimento. Um estudo epidemiológico em crianças e jovens de Moçambique. Porto: Faculdade de Ciências do Desporto e de Educação Física, Universidade do Porto, 4970.

17. McMurray R, Harrell J, Bangdiwala S, Gangsky S (1995). Biological and environmental factors influencing the aerobic power of children. Med Exerc Nutr Health 4: 243-250.

18. Moreno D, Vasconcelos O (2003). Motor performance and maturational status. Study in children of two different school environments (rural and urban). Rev Port Ciências Desporto (Supl) 3 (2): 163-165.
19. Neto C (2001). Aprendizagem, desenvolvimento e jogo de actividade física. In: M. Guedes (Ed). Aprendizagem motora. Problemas e contextos. Lisboa: FMH, 193-220.

20. O’Brien M, Jones D, Rustin M (2000). Children's independent spatial mobility in the urban public realm. Childhood 7: 257-277.

21. Pissara M (1993). Desenvolvimento motor e envolvimento social. Estudo do crescimento e desenvolvimento das capacidades motoras das crianças dos 7 aos 9 anos de idade nos meios rural e urbano. Dissertação de Mestrado. Lisboa: FMH.

22. Reyes M, Tan S, Malina R (2003): Urban-Rural contrasts in the physical fitness of school children in Oxaca, Mexico. Am J Hum Biol 15: 800-813.

23. Rutenfranz J, Anderson L, Seliger V, Masironi R. (1982). Health standards in terms of exercise fitness of schoolchildren in urban and rural areas in various European countries. Ann Clin Res 14(Suppl.), 34: 33-36.

24. Saranga S, Prista A, Maia J (2002). Mudanças nos níveis de aptidão física em função de alterações políticas e sócioeconómicas de 1992-1999. In: A. Prista, J. Maia, S. Saranga, A. Marques (Eds). Saúde, crescimento e desenvolvimento. Um estudo epidemiológico em crianças e jovens de Moçambique. Porto: Faculdade de Ciências do Desporto e de Educação Física, Universidade do Porto, 71-87.

25. Serra M (1992). Desenvolvimento motor, jogo e contexto cultural: estudo comparative da actividade lúdica e do comportamento motor de três grupos de crianças com 6, 7, 8 e 9 anos pertencentes a meios socioculturais diferenciados. Dissertação de Mestrado. Lisboa: FMH.

26. Shrout, P, Fleiss, J (1979). Intraclass correlations: Uses in assessing rater reliability. Psychological Bulletin 86 (2): 420428.

27. Silva M, Sobral F, Malina R (2003). Determinância sociogeográfica da prática desportiva na adolescência. Coimbra: Centro de Estudos do Desporto Infanto-Juvenil, Faculdade de Ciências do Desporto e de Educação Física, Universidade de Coimbra.

28. Sunnegardt J, Bratteby L, Sjodin S (1985). Physical activity and sports involvement in 8- and 13-year-old children in Sweden. Acta Paediatr Scand 74: 904-912.

29. Wilczewski A, Sklad M, Krawczyk B, Saczuk J, Majle B (1996). Physical development and fitness of children from urban and rural areas as determined by Eurofit Test Battery. Biol Sport 13 (2): 113-126. 